\title{
Atenção ao câncer de mama a partir da suspeita na atenção primária à saúde nos municípios de São Paulo e Campinas, Brasil
}

\author{
Breast cancer care from a suspected case detected in primary health \\ care in São Paulo and Campinas, Brazil
}

Cristiane Pereira de Castro (https://orcid.org/0000-0003-1314-8584) ${ }^{1}$

Danila Cristina Paquier Sala (https://orcid.org/0000-0003-3723-6706) ${ }^{2}$

Tereza Etsuko da Costa Rosa (https://orcid.org/0000-0001-9285-0472) ${ }^{3}$

Oswaldo Yoshimi Tanaka (https://orcid.org/0000-0002-5653-0794) ${ }^{1}$
${ }^{1}$ Departamento de Política, Gestão e Saúde, Faculdade de Saúde Pública, Universidade de São Paulo. Av. Doutor Arnaldo 715, Cerqueira César. 01246904 São Paulo SP Brasil. crispcastro@gmail.com ${ }^{2}$ Departamento de Saúde Coletiva, Escola Paulista de Enfermagem, Universidade Federal de São Paulo. São Paulo SP Brasil.

${ }^{3}$ Instituto de Saúde. São Paulo SP Brasil.
Abstract We aimed to identify factors that interfere in breast cancer (BC) suspected cases detected in Primary Health Care (PHC) that drive the referral to Specialized Care (SC) São Paulo and Campinas, Brazil. We interviewed 664 women (353 from São Paulo and 311 from Campinas) diagnosed with BC, referred to SC from PHC. Multilevel logistic regression analysis was used to the identification of associations between BC suspected cases by PHC and the socioeconomic and cancer care variables. The following showed a significant association: higher schooling level $(O R=0.59$; 95\% CI =0.35-0.98); medical visit payment for cancer-related care $(O R=0.59 ; 95 \% C I=0.36$ 0.96), continued attendance at PHC after beginning treatment at $S C(O R=0.62 ; 95 \% C I=0.41$ $0.95)$; breast examined in PHC before referral to $S C(O R=1.61 ; 95 \% C I=1.10-2.34)$ and first mammography requested in PHC (ECM) (OR = 2.81; 95\% CI =1.95-4.00). The request for mammography and the clinical breast examination is incorporated into the PHC for the early detection of $B C$ in the cities. Continuing the care at the PHC and better socioeconomic conditions, such as having a higher education level and capacity to pay for care, can reduce the likelihood of suspected cases. Key words Breast neoplasms, Early detection of cancer, Primary health care, Unified Health System, Health evaluation
Resumo Buscou-se identificar fatores que interferem na suspeita do câncer de mama (CM) pela atenção primária à saúde (APS) que impulsionam o encaminhamento para a atenção especializada (AE) nos municípios de São Paulo e Campinas. Foram entrevistadas 664 mulheres (353 paulistanas e 311 campineiras) diagnosticadas com CM, encaminhadas para AE pela APS. Utilizou-se a análise de regressão logística multinível para a identificação das associações entre a suspeita do CM pela APS, variáveis socioeconômicas e atenção ao câncer. Mostraram associação significativa: nivel superior de escolaridade $(R C=0,59 ;$ IC 95\% =0,35-0,98); pagamento de consulta médica para atendimento relacionado ao câncer $(R C=0,59$; IC 95\% = 0,36-0,96), continuou atendimento na APS após início do tratamento na $A E(R C=0,62$; IC 95\% = 0,41-0,95); mama examinada na APS (ECM) antes do encaminhamento para a AE (RC $=1,61$; IC 95\% = 1,10-2,34) e primeira mamografia solicitada na APS ( $R C=2,81$; IC 95\% = 1,95-4,00). A solicitação da mamografia e o ECM são ações que têm sido incorporadas na APS para a detecção precoce do CM nos municípios. Continuar o atendimento na APS e melhores condições socioeconômicas, como ter nível superior de escolaridade e capacidade de pagar por atendimento, reduzem a chance da suspeita.

Palavras-chave Neoplasias da mama, Detecção precoce de câncer, Atenção primária à saúde, Sistema Único de Saúde, Avaliação em saúde 


\section{Introdução}

O aumento das doenças não transmissíveis em consequência do intenso envelhecimento da população e à incorporação de tecnologias de saúde vem ocasionando um grande impacto na organização dos sistemas de saúde. A complexidade da gestão das condições crônicas deve garantir a detecção precoce e o tratamento, em particular, do câncer ${ }^{1-3}$.

A mortalidade por câncer em geral segue crescente em todo o mundo, o câncer de mama (CM) apresenta uma situação específica de diminuição nos países desenvolvidos, como os do Norte e Oeste da Europa ${ }^{4}$. Em países em desenvolvimento, como o Brasil, observa-se situação inversa, em decorrência da presença de fatores comportamentais, barreiras socioculturais e dificuldades de acesso a serviços de saúde para detecção precoce, elucidação diagnóstica e tratamento $^{5-7}$.

Estatísticas globais apontam que o CM é o segundo de maior incidência considerando ambos os sexos, e no caso das mulheres a neoplasia tem maior ocorrência e mortalidade ${ }^{8}$. No Brasil, o Instituto Nacional de Câncer José Alencar Gomes da Silva (INCA) prevê 59.700 casos novos de CM para o biênio 2018-2019, com um risco estimado de 56.33 casos a cada 100 mil mulheres9. Considerando o locus de investigação deste artigo, a previsão em 2018 para o estado de São Paulo era de 16.340 casos $^{9}$, sendo que na região Sudeste o risco de morrer devido ao $\mathrm{CM}$ vem diminuindo progressivamente nas mulheres nascidas após a década de $1930^{10}$.

$\mathrm{O}$ acesso universal da população ao SUS tem sido desafiado pela heterogeneidade na implementação do sistema, pela presença de iniquidades na oferta de serviços de saúde e a insuficiência nas ações de cuidado ${ }^{11-13}$.

No que se refere à atenção ao $\mathrm{CM}$, preconizam-se ações de promoção, prevenção, detecção precoce, tratamento oportuno e cuidados paliativos integrados e continuados pelos serviços da rede primária de saúde ${ }^{12,14-18}$.

Entre tais ações na APS, tem-se enfatizado a detecção precoce do CM, não apenas pela importância da realização de etapas iniciais relacionadas à identificação de sinais e sintomas do câncer, mas por sua capacidade na coordenação do cuidado, inclusive no que se refere à regulação assistencial dos serviços da AE nos casos suspeitos ${ }^{19,20}$.

Atualmente, são consideradas ações para a detecção precoce do câncer de mama no Brasil o rastreamento mamográfico entre mulheres assintomáticas na faixa etária de 50 a 69 anos a cada dois anos e o diagnóstico precoce, a partir do exame clínico das mamas e/ou mamografia diagnóstica entre mulheres sintomáticas em qualquer idade ${ }^{21}$.

O crescente papel da APS na efetivação da linha de cuidado oncológica decorre da maior capacidade de conhecer o território, organizar ações de rastreio, identificar casos suspeitos e viabilizar a melhor trajetória dentro do sistema de saúde, proporcionando elucidação diagnóstica em menor tempo e mais qualidade a cada uma dessas etapas ${ }^{22,23}$.

Mas é preciso atenção e constante aprimoramento das ações de deteç̧ão precoce, visto que sua ocorrência em desacordo com as recomendações do Ministério da Saúde (MS) pode comprometer sua efetividade e implicar maior risco às mulheres ${ }^{24}$.

Diante do exposto, o objetivo deste estudo é identificar fatores que interferem na suspeição de CM pela atenção primária a saúde que impulsionam o encaminhamento para a atenção especializada nos municípios de São Paulo e Campinas.

\section{Métodos}

Foram analisados dados parciais do projeto "Análise Multifacetada sobre o Acesso a partir da Atenção Primária à Saúde, o funcionamento e a utilização da Atenção Especializada para quatro condições traçadoras em quatro grandes cidades brasileiras", que recebeu financiamento por meio do edital do MCTI/CNPq/MS/SCTIE e seguiu as normas éticas de pesquisa com seres humanos $\mathrm{e}$ aprovação no CEP.

No presente artigo, o $\mathrm{CM}$ é a condição traçadora analisada nos municípios de São Paulo e Campinas. Segundo Kessner (1973), a escolha da condição traçadora como fenômeno que contribui nos estudos de avaliação deve ser orientada por alguns critérios, como: ser um problema de saúde de alta prevalência, com definição diagnóstica e padrões determinados para o manuseio do paciente, em que possíveis intervenções conduzam a impacto no curso da doença ${ }^{25}$.

Adotou-se o papel privilegiado da APS para identificar sinais e sintomas do CM e para impulsionar a mulher com a suspeição no percurso da linha de cuidado do CM, proposto pela Política Nacional para Prevenção e Controle do Câncer (PNPCC-RAS) $)^{16}$. Entretanto, estudos apontam que a suspeição de $\mathrm{CM}$ ocorre em diferentes serviços assistenciais que compõem a oferta em 
cada território ${ }^{24}$. Levantou-se a seguinte questão: o que estaria levando a mulheres identificadas na linha de cuidado do CM do SUS a não terem feito a suspeição do CM na APS?

Foram incluídas mulheres com diagnóstico confirmado de CM e que obtiveram acesso ao serviço de oncologia pela APS por meio do sistema de regulação SUS municipal. Foram inelegíveis para participar do estudo mulheres em processo de elucidação diagnóstica. Essas informações foram obtidas pelas respostas afirmativas, respectivamente, para as seguintes perguntas: "O diagnóstico é CM?"; "Veio para este serviço encaminhada por uma unidade básica de saúde, posto de saúde ou centro de saúde?”. A fase do tratamento em que se encontravam as mulheres no dia da entrevista não foi condição de inclusão ou exclusão no estudo.

As entrevistas foram conduzidas na $\mathrm{AE}$, que são serviços ambulatoriais com especialistas em mastologia e/ou oncologia, inseridos em unidades hospitalares de alta complexidade.

Em São Paulo, o local de desenvolvimento da pesquisa foi o Instituto Sírio Libanês (ISL), instituição de direito privado, prestadora de serviço público na rede SUS. Esse serviço, por meio do Programa de Desenvolvimento Institucional do Sistema Único de Saúde (PROADI - SUS) estava inserido na linha de cuidado, ofertando, à época da pesquisa, 70 vagas/mês para atendimento da população usuária do SUS ${ }^{26}$.

Em Campinas, a pesquisa foi desenvolvida nos Ambulatórios de Oncologia do Hospital Municipal Dr. Mario Gatti e no Centro de Atenção Integral à Saúde da Mulher ${ }^{27}$, uma das unidades hospitalares pertencentes à Faculdade de Ciências Médicas da Universidade Estadual de Campinas.

Todo o processo de amostragem, coleta de dados e planos de análise podem ser consultados na página eletrônica da pesquisa ACESSUS (https://www.fcm.unicamp.br/acessus/metodologia/inquerito-amostragem/plano-de-amostragem/cancer-de-mama).

As entrevistas foram realizadas entre março e junho de 2016. Utilizou-se questionário, elaborado exclusivamente para esta pesquisa, contendo 52 questões fechadas, que visaram à identificação e à verificação de eventos referentes à trajetória das mulheres nos serviços da APS e da AE, com base nas recomendações oficiais da área ${ }^{16,19,21}$. Tal questionário pode ser consultado na página eletrônica da pesquisa ACESSUS (https://www.fcm. unicamp.br/acessus/metodologia/inquerito-amostragem/questionarios/cancer-de-mama).

\section{Análise dos dados}

O quadro referencial para analisar a atenção ao CM foi construído conforme as recomendações da PNPCC-RAS ${ }^{16}$, com foco no papel da APS como coordenadora das redes de atenção e com a função de promover acesso do usuário do SUS à AE a partir das ações de detecção precoce do câncer.

Levando-se em consideração as especificidades de organização da APS nos diferentes municípios e a diversidade de características individuais das mulheres, optou-se por utilizar o modelo de regressão logística multinível ${ }^{28}$, com o nível contextual representado pelo município.

Adotou-se como variável dependente a "suspeita do CM pela APS", coletada a partir da seguinte pergunta: "A sra. recebeu pela primeira vez a informação sobre a suspeita ou diagnóstico de CM no Posto ou Centro de Saúde?” Partimos do pressuposto que a identificação de mulheres com suspeita de câncer de mama é uma competência da APS, a fim de efetivar a detecção precoce normatizada pela linha de cuidado oncológica ${ }^{19}$. Obter tal informação representa o passo inicial para a elucidação diagnóstica, configurando-se, portanto, como uma atribuição fundamental nesse nível de atenção para alcançar o cuidado integral ${ }^{19}$. É importante esclarecer que o questionário não possibilitou explorar se a suspeita ou o diagnóstico do câncer pela APS foi decorrente de uma conduta profissional, determinação do protocolo municipal, consequência de ações de rastreamento ou investigação diagnóstica de sinais ou sintomas.

Em nível individual, as variáveis independentes relativas às condições socioeconômicas selecionadas para o estudo foram: idade (anos), escolaridade, cor da pele, possui plano de saúde/ou convênio médico e pagou consulta médica para atendimento relacionado ao câncer de mama. Já as variáveis relacionadas à atenção ao câncer foram: continuou atendimento na APS após início do tratamento na AE, mama examinada na APS antes do encaminhamento para $\mathrm{AE}$, primeira mamografia solicitada na APS e primeira mamografia feita no SUS.

Para verificar as associações das variáveis independentes com a suspeita do câncer na APS, estabeleceu-se o valor de p inferior a 0,05 na análise univariada como elegível para compor o modelo multivariado.

Na primeira etapa de modelagem, foi ajustado o modelo nulo na regressão logística multinível, que inclui apenas o intercepto aleatório, e a 
análise gráfica caterpillar dos resíduos dos municípios.

$\mathrm{Na}$ etapa seguinte, foram ajustados modelos multinível univariados para avaliar cada uma das variáveis do nível dos indivíduos, analisando-se a variância explicada pelo efeito do município e a associação delas com o desfecho.

$\mathrm{Na}$ terceira etapa, ajustou-se o modelo multivariado com a inclusão das variáveis do nível individual que mantiveram o nível de significância do critério estabelecido na análise univariada. Além disso, previamente ao ajuste do modelo multivariado final, foram feitas as análises de multicolinearidade entre as variáveis independentes, por meio do cálculo do fator de inflação de variância (VIF). Vale ressaltar que as variáveis idade, escolaridade e "continuou atendimento na APS" foram consideradas variáveis de controle no modelo final. As análises foram conduzidas no pacote Statistical Software for Professional (STATA), versão 10.

\section{Resultados}

Compuseram a casuística 664 mulheres, das quais 353 e 311 foram entrevistadas, respectivamente, nos municípios de São Paulo e Campinas, todas com diagnóstico de CM confirmado, encaminhadas ao serviço especializado pela APS.

A Tabela 1 mostra a distribuição das variáveis socioeconômicas e daquelas relacionadas às etapas da atenção ao CM selecionadas para análise. Como esperado, trata-se de uma população de mulheres de 50 anos ou mais $(72,9 \%)$. Aproximadamente metade $(48,0 \%)$ tinha frequentado apenas até o ensino fundamental, no entanto $17,3 \%$ responderam ter nível superior de escolaridade. Em que pese o predomínio de mulheres que disseram ser de cor/raça branca $(60,5 \%)$, surpreendeu a prevalência de mulheres pretas $(10,8 \%)$. As frequências das entrevistadas que referiram ter plano de saúde e que pagaram consulta médica por atendimento ao câncer foram, respectivamente, $11,1 \%$ e $17,2 \%$.

Após o início do tratamento na AE, menos de um terço das mulheres continuou o atendimento na APS (26,2\%). Já em relação às etapas de atenção do CM antes do início do tratamento, a APS teve papel importante para a maioria das entrevistadas, visto que $68,3 \%$ e $54,2 \%$ delas declararam ter sido esse o serviço em que realizaram exame clínico das mamas antes do encaminhamento à $\mathrm{AE}$, onde tiveram a primeira mamografia solicitada, respectivamente. Além disso, confirmando o perfil socioeconômico de baixo poder aquisitivo (não ter plano de saúde ou não ter pago por consulta médica), 78,3\% das mulheres responderam ter feito a primeira mamografia no SUS.

O modelo nulo e a análise gráfica caterpillar dos resíduos indicaram a existência do efeito do contexto municipal sobre o desfecho em estudo, resultado que orientou a escolha da técnica estatística multinível como a mais adequada para a obtenção de estimativas das razões de chance (RC) mais precisas.

A Tabela 2 apresenta os modelos de regressão logística multinível para a suspeita de câncer de mama na APS. A variância do intercepto do modelo nulo indicou que os municípios explicam 7\% da variabilidade de suspeita de câncer de mama na APS, ou seja, existe uma heterogeneidade entre São Paulo e Campinas no que diz respeito a esse procedimento. Na segunda coluna, são apresentados os modelos multinível univariados com a inclusão das variáveis individuais. As variâncias apresentadas nos respectivos modelos, no geral indicam que, quando incluídas individualmente, a variância explicada pelo efeito dos municípios aumenta. No caso da primeira mamografia solicitada pela APS, essa variância aumentou de 7\%, no modelo nulo, para 15\% quando essa variável foi incluída.

A presença de multicolinearidade (VIF maiores e próximos de 1 - dados não apresentados) indicou ausência de efeito importante de interação entre as variáveis independentes. Dessa forma, o modelo multivariado final foi composto pelas variáveis estatisticamente significativas nas análises univariadas e multivariada, ao nível de $5 \%$, bem como pelas variáveis de controle do nível dos indivíduos. Da mesma forma que os modelos univariados, pelo aumento da magnitude da variância do modelo ajustado em relação ao modelo nulo (de 0,07 para 0,13 ), pode-se concluir que a variância explicada pelo efeito dos municípios aumentou a variabilidade da suspeita de câncer de mama na APS nos municípios.

O modelo ajustado final apontou que ter a mama examinada na APS antes do encaminhamento para AE e ter a primeira mamografia solicitada na APS, em comparação com as situações opostas, aumentam significativamente as chances de ter a suspeita do CM na APS. No entanto, ter cursado ensino superior, ter pago consulta médica para atendimento relacionado ao $\mathrm{CM}$ e continuar o atendimento na APS após o início do tratamento na $\mathrm{AE}$, em relação às respectivas condições contrárias, diminuem significativamente a chance de ter a suspeita do CM na APS. 
Tabela 1. Distribuição das variáveis socioeconômicas e das etapas de atenção ao câncer de mama referidas pelas mulheres em tratamento nos serviços de alta complexidade em oncologia, São Paulo e Campinas, 2016.

\begin{tabular}{|c|c|c|}
\hline Variáveis & $\mathbf{N}$ & $\%$ \\
\hline \multicolumn{3}{|l|}{ Idade (anos) } \\
\hline $19-49$ & 180 & 27,1 \\
\hline $50+$ & 484 & 72,9 \\
\hline \multicolumn{3}{|l|}{ Escolaridade } \\
\hline Analfabeto & 43 & 6,5 \\
\hline Ensino fundamental & 319 & 48,0 \\
\hline Ensino médio & 187 & 28,2 \\
\hline Ensino superior & 115 & 17,3 \\
\hline \multicolumn{3}{|l|}{ Cor da pele } \\
\hline Branca & 397 & 60,5 \\
\hline Preta & 71 & 10,8 \\
\hline Parda & 166 & 25,3 \\
\hline Amarela & 22 & 3,4 \\
\hline \multicolumn{3}{|l|}{ Possui plano de saúde e/ou convênio médico } \\
\hline Não & 590 & 88,9 \\
\hline Sim & 74 & 11,1 \\
\hline \multicolumn{3}{|c|}{ Pagou consulta médica para atendimento relacionado ao câncer } \\
\hline Não & 549 & 82,8 \\
\hline Sim & 114 & 17,2 \\
\hline \multicolumn{3}{|c|}{ Continua atendimento na APS após início do tratamento na AE } \\
\hline Não & 488 & 73,8 \\
\hline Sim & 173 & 26,2 \\
\hline \multicolumn{3}{|c|}{ Mama examinada na APS antes do encaminhamento para AE } \\
\hline Não & 196 & 31,7 \\
\hline Sim & 422 & 68,3 \\
\hline \multicolumn{3}{|l|}{ Primeira mamografia solicitada na APS } \\
\hline Não & 294 & 45,8 \\
\hline Sim & 348 & 54,2 \\
\hline \multicolumn{3}{|l|}{ Fez a primeira mamografia no SUS } \\
\hline Não & 139 & 21,7 \\
\hline Sim & 502 & 78,3 \\
\hline
\end{tabular}

Fonte: Autores.

As mulheres com 50 anos ou mais se mostraram associadas à suspeita do CM na APS somente na análise univariada, revelando que a idade não é independente das outras variáveis individuais. A escolaridade mostrou associação significativa apenas para a categoria nível superior, tanto na análise univariada quanto na ajustada, apresentando nesta chances $41 \%$ menores de ter a suspeita do CM na APS ( $\mathrm{RC}=0,59$; IC 95\%: $0,35-0,98 ; \mathrm{p}=0,04)$, em relação àquelas sem nenhuma escolarização.

No que se refere à cor da pele autorreferida pelas mulheres do estudo, identificou-se que tal variável não apresentou associação estatisticamente significativa, ou seja, a cor da pele não se configura como uma variável explicativa para a suspeita de câncer pela APS nos municípios estudados.
Com relação aos indicadores de poder aquisitivo das mulheres, no modelo ajustado, somente a variável "pagou consulta médica para atendimento relacionado ao câncer" manteve associação estatisticamente significativa, embora a magnitude da $\mathrm{RC}$ e a força da associação tenham sido atenuadas ( $\mathrm{RC}=0,59$; IC 95\%: 0,36-0,96; $\mathrm{p}=$ $0,03)$. Quanto a possuir plano de saúde, a análise de multicolinearidade não indicou necessidade de testar o efeito da interação com outras variáveis independentes.

A variável "continuou atendimento na APS após início do tratamento na AE" não mostrou associação significativa no modelo univariado nem efeito de interação com outras variáveis, no entanto ela foi mantida como controle no modelo ajustado final, em razão de sua importância na completude do desenho do presente estudo. No modelo ajusta- 
Tabela 2. Associações entre variáveis individuais e contextual, determinadas por análise de regressão logística multinível, São Paulo e Campinas, 2016.

\begin{tabular}{|c|c|c|c|c|c|c|c|c|}
\hline \multirow{2}{*}{ Variáveis } & \multirow{2}{*}{$\begin{array}{l}\text { Modelo } \\
\text { nulo }\end{array}$} & \multicolumn{3}{|c|}{ Modelos univariados } & \multirow[b]{2}{*}{$\sigma_{u}^{2}$} & \multicolumn{3}{|c|}{ Modelo multivariado final } \\
\hline & & RC & IC $(95 \%)$ & p-valor & & RC & IC (95\%) & p-valor \\
\hline Idade (anos) & & & & & 0,08 & & & \\
\hline $19-49$ & & 1,00 & & & & 1,00 & & \\
\hline $50+$ & & 1,63 & $1,15-2,32$ & $<0,01$ & & 1,39 & $0,93-2,09$ & 0,11 \\
\hline Escolaridade & & & & & 0,10 & & & \\
\hline Analfabeto & & 1,00 & & & & 1,00 & & \\
\hline Fundamental e médio & & 0,64 & $0,34-1,24$ & 0,18 & & 0,85 & $0,56-1,28$ & 0,43 \\
\hline Superior & & 0,35 & $0,17-0,73$ & $<0,01$ & & 0,59 & $0,35-0,98$ & 0,04 \\
\hline Cor da pele & & & & & 0,07 & & & \\
\hline Branca & & 1,00 & & & & * & & \\
\hline Preta & & 0,78 & $0,47-1,30$ & 0,33 & & & & \\
\hline Parda & & 1,19 & $0,82-1,72$ & 0,35 & & & & \\
\hline Amarela & & 0,98 & $0,41-2,33$ & 0,96 & & & & \\
\hline $\begin{array}{l}\text { Possui Plano de saúde e/ou } \\
\text { convênio médico }\end{array}$ & & & & & 0,08 & & & \\
\hline Não & & 1,00 & & & & * & & \\
\hline Sim & & 0,57 & $0,34-0,93$ & 0,03 & & & & \\
\hline $\begin{array}{l}\text { Pagou consulta médica para } \\
\text { atendimento relacionado ao } \\
\text { câncer de mama }\end{array}$ & & & & & 0,06 & & & \\
\hline Não & & 1,00 & & & & 1,00 & & \\
\hline Sim & & 0,43 & $0,28-0,65$ & $<0,01$ & & 0,59 & $0,36-0,96$ & 0,03 \\
\hline $\begin{array}{l}\text { Continuou atendimento na APS } \\
\text { após início do tratamento na AE }\end{array}$ & & & & & 0,06 & & & \\
\hline Não & & 1,00 & & & & 1,00 & & \\
\hline Sim & & 0,91 & $0,62-1,34$ & 0,64 & & 0,62 & $0,41-0,95$ & 0,03 \\
\hline $\begin{array}{l}\text { Mama examinada na APS antes do } \\
\text { encaminhamento para AE }\end{array}$ & & & & & 0,09 & & & \\
\hline Não & & 1,00 & & & & 1,00 & & \\
\hline Sim & & 1,56 & $1,11-2,21$ & 0,01 & & 1,61 & $1,10-2,34$ & 0,01 \\
\hline $\begin{array}{l}\text { Primeira mamografia solicitada na } \\
\text { APS }\end{array}$ & & & & & 0,15 & & & \\
\hline Não & & 1,00 & & & & 1,00 & & \\
\hline Sim & & 3,48 & $2,48-4,89$ & $<0,01$ & & 2,81 & $1,95-4,00$ & $<0,001$ \\
\hline Primeira mamografia feita no SUS & & & & & 0,09 & * & & \\
\hline Não & & 1,00 & & & & & & \\
\hline Sim & & 2,10 & $1,43-3,10$ & $<0,01$ & & & & \\
\hline 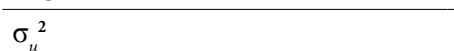 & 0,07 & & & & & 0,13 & & \\
\hline $\begin{array}{l}\text { Modelo multivariado final - manutenção d } \\
\text { tratamento na AE como controles. }{ }^{*} \text { Variáve } \\
\text { municípios. RC: razão de chances; IC: inter } \\
\text { Sistema Único de Saúde. }\end{array}$ & $\begin{array}{l}\text { las variáveis } \\
\text { el excluída d } \\
\text { rvalo de con }\end{array}$ & $\begin{array}{l}\text { escolar } \\
\text { lo mode } \\
\text { fiança; }\end{array}$ & $\begin{array}{l}\text { dade, idade } \\
\text { o final devic } \\
\text { E: atenção e }\end{array}$ & $\begin{array}{l}\text { continuou } \\
\text { ao valor } \\
\text { pecializad }\end{array}$ & $\begin{array}{l}\text { tendi } \\
p>0 \\
\text { APS: }\end{array}$ & $\begin{array}{l}\text { ento na } \\
5 . \sigma_{u}{ }^{2} \mathrm{Y} \\
\text { ençâo p }\end{array}$ & $\begin{array}{l}\text { APS após iní } \\
\text { ariância expl } \\
\text { imária à saú }\end{array}$ & $\begin{array}{l}\text { do } \\
\text { ada pelos } \\
\text {; SUS: }\end{array}$ \\
\hline
\end{tabular}

do, foi surpreendente que essa variável mostrou associação estatisticamente significativa, sendo que a chance daquelas que continuaram atendimento na
APS após início do tratamento na AE foi 38\% menor de obter a suspeita de CM na APS $(\mathrm{RC}=0,62$; IC 95\%: 0,41-0,95; $\mathrm{p}=0,03$ ). 
A análise univariada dá indicações de que as mulheres que tiveram a mama examinada na APS antes do encaminhamento para a AE tinham chance $56 \%$ maior de ter a suspeita de CM nesse serviço (RC = 1,56; IC 95\%: 1,11-2,21; $p=0,01$ ). Esses resultados foram mantidos mesmo após o ajuste do modelo ( $\mathrm{RC}=1,61$; IC 95\%: 1,10-2,34; $\mathrm{p}=0,01$ ), o que aponta a importância dessa variável, independente das demais para explicar o desfecho.

Observa-se que a variável "primeira mamografia solicitada na APS" se configurou na de maior magnitude no valor da RC e com forte associação com a suspeita de câncer na APS, tanto na análise univariada ( $\mathrm{RC}=3,48$; IC 95\%: 2,48$4,89 ; \mathrm{p}<0,01)$ quanto no modelo ajustado $(\mathrm{RC}=$ 2,81; IC 95\%: 1,95-4,00; $p<0,001)$. Esses resultados indicam que as mulheres que fizeram a primeira mamografia por solicitação da APS tinham chances, aproximadamente, três vezes maiores de suspeita de câncer de mama realizada na APS, independentemente de outras variáveis.

A análise de regressão univariada apontou que fazer a mamografia no SUS aumenta a chance em cerca de duas vezes de obter a suspeita de câncer na APS ( $\mathrm{RC}=2,10$; IC 95\%: 1,43-3,10; $\mathrm{p}$ $<0,01)$. Não obstante, no modelo ajustado ela não mostrou efeito de interação com as outras variáveis e não manteve a significância ao nível de $5 \%$, tendo sido retirada do modelo multivariado final.

\section{Discussão}

Historicamente, no Brasil, esforços têm sido dedicados à implementação de políticas públicas para o acesso da população às ações de detecção precoce do $\mathrm{CM}$. As recomendações envolvendo o rastreamento e o diagnóstico precoce desse agravo vêm sendo atualizadas, e a suspeita junto às mulheres, por parte dos serviços da APS, se configura como etapa importante da linha de cuidado para o $\mathrm{CM}^{31}$.

Neste estudo, pôde-se constatar que as recomendações ligadas à detecção precoce por meio da suspeição do CM, seja via solicitação de mamografia ou pelo exame clínico das mamas, vêm sendo incorporadas. No entanto, nossos resultados também indicam que, além dessas etapas clínicas, as características individuais das mulheres e do contexto influenciam nesse processo.

A idade igual ou superior a 50 anos aumenta a chance da primeira suspeição no serviço de APS nos municípios. No entanto, observou-se que não houve manutenção da significância estatística no modelo ajustado, indicando que não é independente de outras variáveis. Tal fato pode ser decorrente da heterogeneidade entre o município de Campinas e de São Paulo quanto ao cumprimento das recomendações para o grupo etário em relação à indicação do exame mamográfico como estratégia de rastreio para detecção precoce de CM. Discutiremos o tema mais adiante.

Diversos estudos têm documentado a existência de desigualdades raciais que se manifestam pela dificuldade de acesso da mulher negra ao exame de mamografia $a^{32}$ è unidade oncológi$\mathrm{ca}^{33,34}$, bem como pela chance duplicada de apresentar pior prognóstico em decorrência da maior proporção de estágios avançados no diagnóstico do câncer, em comparação com as mulheres brancas ${ }^{35}$. O presente estudo, em concordância com esses achados, evidenciou que, entre as mulheres de cor preta, a chance de ter a suspeita de CM na APS era reduzida em relação às brancas, em que pese a fraca associação encontrada.

Análises de itinerários terapêuticos utilizados por mulheres com CM indicaram que aquelas com menor renda e escolaridade utilizam mais frequentemente a APS como porta de entrada, ao contrário daquelas com maior renda, que acessam habitualmente os serviços privados ${ }^{36}$. Os achados do presente estudo corroboram a tendência de redução na chance de suspeita de câncer pela APS conforme aumenta a escolaridade das mulheres.

No que se refere às etapas da atenção ao CM, a realização da mamografia no SUS, a solicitação desse exame pela APS e o exame clínico das mamas são variáveis associadas à maior suspeita de CM pela APS nos municípios estudados.

O rastreamento do CM por meio do exame clínico das mamas fez parte das recomendações ministeriais de 2004 até 2015. Atualmente, sua prática vem sendo retomada, em conjunto com a anamnese, como estratégia fundamental para a identificação de sinais e sintomas suspeitos do CM. Tais ações favoreceriam o acesso das usuárias sob risco à investigação diagnóstica na $\mathrm{AE}^{19}$. O ECM mostrou associação estatisticamente significativa com a suspeição de CM na APS, mesmo no modelo ajustado. Esse achado mostra que o uso do ECM nos municípios não foi abandonado entre as práticas para a detecção precoce do CM e corrobora a sua utilização como prática do exame físico e estratégia na identificação de sinais e sintomas. Essa situação pode ser confirmada em artigo de revisão que apontou o ECM como 
uma das ações de maior prevalência no país para detecção precoce do $\mathrm{CM}^{37}$, devido ao fácil acesso, baixo custo e à relevância na identificação de usuárias sob risco. Ainda assim, outros estudos indicam que o ECM não é realizado pela maioria dos profissionais da rede primária devido ao pouco conhecimento de suas etapas propedêuti$\mathrm{cas}^{38,39} \mathrm{e}$ à falta de tempo e de local apropriado ${ }^{40}$.

Em relação ao exame de mamografia, as mulheres que tiveram a solicitação pela APS apresentaram aproximadamente três vezes mais chances de ter a suspeita do agravo, mesmo ajustando por outras variáveis. Portanto, a mamografia como uma ação voltada à detecção precoce do $\mathrm{CM}$ parece irrefutavelmente incorporada ao processo de trabalho dos profissionais da rede primária.

A solicitação da mamografia como exame de rastreio é identificada como um procedimento chave para a entrada da mulher na rede de atenção oncológica pela $\mathrm{APS}^{14,16}$. Os resultados deste estudo estão em consonância com pesquisa realizada no país que constatou a ampliação do acesso à mamografia de forma compatível com o aumento na oferta desse exame tanto na rede pública quanto na conveniada ${ }^{41}$, e a maior densidade de mamógrafos nos estados de São Paulo, entre outros ${ }^{42}$.

Entretanto, cabe chamar atenção para uma falta de convergência entre as recomendações ministeriais e as da sociedade médica em relação à indicação do exame mamográfico como estratégia de rastreio para a detecção precoce do CM. Por um lado, o MS alterou a população-alvo elegível para o exame de 40 até os 69 anos para 50 a 69 anos, com periodicidade bienal ${ }^{19,21}$. Por outro, a Sociedade Brasileira de Mastologista, respaudada pela Lei $n^{\circ} 11.664 / 08^{43}$, tem mantido a recomendação de mamografia de rastreio a partir dos 40 anos, com periodicidade anual. Tal situação pode trazer ambiguidade na interpretação de para quem, por que e quando deve-se solicitar o exame mamográfico, dificultando a tomada de decisão pelo profissional de saúde da APS e a utilização do serviço pelas usuárias.

Estudo apontou que, no Brasil, quase 40\% das mamografias de rastreamento estão em desacordo com a faixa etária recomendada, e tal descompasso pode estar intensificando o estrangulamento da capacidade diagnóstica da rede SUS, agregando exposição das mulheres aos efeitos do sobrediagnóstico e, por consequência, ao sobretratamento ${ }^{44}$. Deve-se reconhecer, ainda, que se soma ao contexto descrito o desconhecimento dos profissionais como motivo para o não seguimento dessas recomendações ${ }^{37,40}$, bem como para a não implementação de uma linha de cuidado de CM com ações de rastreio organizadas na rede de atenção oncológica ${ }^{45}$. Esse contexto pode ser uma das explicações dos resultados do presente estudo quanto à ausência de associação da variável idade com a suspeita de CM na APS, comentada anteriormente.

Outra variável analisada no presente estudo foi a continuidade da atenção na APS após o início do tratamento na $\mathrm{AE}$ como indicador de vínculo ou de longitudinalidade do cuidado na $\mathrm{APS}^{46}$. Entretanto, essa variável só mostrou associação com a suspeita de câncer identificada na APS no modelo final, indicando que o efeito dela advém da relação com as demais variáveis. Justamente as mulheres que continuaram atendimento na APS após início do tratamento na $\mathrm{AE}$ tiveram menor chance de ter obtido a suspeita de CM na APS. Ou seja, o fato de a APS dar início ao seguimento na linha de cuidado do CM não garante o vínculo da mulher com esse ponto de atenção à saúde. É possível que outras razões levem algumas mulheres a retornarem à APS após o diagnóstico do $\mathrm{CM}$, e não propriamente a continuidade do cuidado para o agravo em si. No contexto de doenças graves como o câncer, que requerem tratamentos complexos e a longo prazo, em geral o vínculo paciente-serviço é estabelecido onde é realizado o tratamento, estimulado pela falta de contrarreferência como dificultadora da coordenação do cuidado pela $\mathrm{APS}^{47}$.

Outras evidências, não obstante, mostram a importância da capacidade da APS em dar continuidade no vínculo estabelecido com pacientes portadoras de CM, no sentido de produzir a integralidade da atenção ${ }^{48}$. Isso atesta o papel destacado da APS, por sua facilidade no estabelecimento de vínculo com as mulheres e na efetividade da linha de cuidado na rede de atenção oncológica $^{49}$.

Provavelmente, a heterogeneidade entre os municípios identificada pela análise multinível tem relação com os modelos de APS desenvolvidos nos respectivos locais, os quais favorecem ou não determinados atributos desses serviços.

No município de São Paulo, observa-se uma rede primária com expansão da estratégia de saúde da família, mas que segue em transformação, sendo, ainda, composta por distintos arranjos, tais como unidades básicas tradicionais, Estratégia de Saúde da Família exclusiva, atendimento médico ambulatorial e unidades mistas e/ou integradas ${ }^{50}$.

Em Campinas, contudo, a instituição da APS foi iniciada na década de 1970, com apoio das 
universidades locais ${ }^{51}$. Em 1990, implantou-se o modelo de atenção em defesa da vida na rede primária, valorizando a incorporação de tecnologias leves, como acolhimento e presença de equipes multiprofissionais ampliadas ${ }^{52}$. E no início de 2000, a constituição do Projeto Paideia ${ }^{53,54}$, vigente até o período da pesquisa, manteve uma rede de APS com ampliação das equipes, incluindo as recomendações da Estratégia de Saúde da Família. Ou seja, num contexto de doenças cujo alcance da integralidade do cuidado é bastante complexa, como no caso do CM, um modelo de APS, que estabelece vínculo com suas usuárias, pode ter maior efetividade na linha de cuidado na rede oncológica ${ }^{20,36}$.

Uma das limitações deste estudo diz respeito à obtenção dos dados por meio do autorrelato das usuárias, algumas delas já em tratamento há muito tempo, sendo possível gerar viés de informação e/ou de memória. Tendo em vista a natureza das perguntas, mesmo considerando a possível variação das informações prestadas, avaliou-se que o relato de usuárias com CM é capaz de produzir informações sobre acesso e cuidado insubstituíveis.

É importante destacar que não foi escopo do estudo a análise diferenciada entre as mulheres que tiveram o diagnóstico do câncer por meio do rastreamento ou da investigação dos sinais ou sintomas suspeitos. Portanto, o questionário não explorou o contexto em que o diagnóstico do CM foi realizado na APS, considerando-se apenas a sua competência para a detecção precoce do câncer normatizada pela linha de cuidado.

Outra limitação se deve à restrição da população amostrada, principalmente em São Paulo, onde foram entrevistadas mulheres em tratamento num único serviço dos diversos existentes no município. Pelo fato de se tratar de um programa do Proadi-SUS dentro de hospital filantrópico/ privado, é possível ter ocorrido viés de amostra. No entanto, a gestão municipal consultada assegurou que o fluxo para regulação das consultas com oncologista no serviço seguia a pactuação vigente na cidade de São Paulo.

\section{Considerações finais}

A análise dos dados mostrou que a solicitação da mamografia e o ECM são ações que têm sido incorporadas na APS para a detecção precoce do CM nos municípios. Dessa forma, pode-se afirmar que a APS exerce o seu papel de ordenadora da atenção ao CM e dá início ao percurso na linha de cuidado, sem contudo conseguir coordenar o cuidado, pois parece não garantir o vínculo da mulher com esse ponto de atenção à saúde.

A definição do grupo etário, tendo em vista a cobertura da população alvo, é um fator crítico para as ações de detecção precoce do CM, e que merece ser discutido por formuladores de políticas, gestores e profissionais de saúde atuantes em todos os níveis de atenção, bem como por estudiosos e pela sociedade médica. Portanto, há fragilidades nesse processo, sobretudo na organização de uma linha de cuidados que assegure à usuária atenção integral com diagnóstico e tratamento oportuno.

Este trabalho sinaliza a necessidade de novos estudos, que visem ampliar a compreensão e a implementação de políticas públicas de saúde voltadas a condições crônicas, reconhecendo as influências de oferta e consumo de tecnologias, do comportamento do sujeito na busca de saúde e do padrão de utilização dos serviços.

\section{Colaboradores}

Todos os autores contribuíram na elaboração do artigo, sendo que CP Castro trabalhou na concepção, metodologia e redação final; DCP Sala atuou na pesquisa e redação final; TEC Rosa colaborou na metodologia e revisão crítica; OYT na concepção, metodologia e revisão crítica. Todos os autores aprovaram a versão final do trabalho a ser publicada. 


\section{Agradecimentos}

Ao Conselho Nacional de Desenvolvimento Científico e Tecnológico ( $\mathrm{CNPq}$ ) pelo subsídio financeiro e pela bolsa de pós-doutoramento.

\section{Referências}

1. Pou AS, Tumas N, Coquet JB, Niclis C, Román MD, Díaz MP. Burden of cancer mortality and differences attributable to demographic aging and risk factors in Argentina, 1986-2011. Cad Saude Publica 2017; 33(2):1-13.

2. Prince MJ, Wu F, Guo Y, Gutierrez Robledo LM, O'Donnell M, Sullivan R, Yusuf S The burden of disease in older people and implications for health policy and practice. Lancet 2015; 385(9967):549-562.

3. Malta DC, Moura L, Prado RR, Escalante JC, Schmidt MI, Duncan BB. Mortalidade por doenças crônicas não transmissíveis no Brasil e suas regiões, 2000 a 2011. Epidemiol Serv Saude 2014; 23(4):599-608.

4. Carioli G, Malvezzi M, Rodrigues T, Bertuccio P, Negri E, La Vecchia C. Trends and predictions to 2020 in breast cancer mortality in Europe. The Breast 2017; 36:89-95.

5. Gonçalves LLC, Travassos GL, Almeida AM, Guimarães AMDN, Gois CFL. Barreiras na atenção em saúde ao câncer de mama: percepção de mulheres. Rev Esc Enferm 2014; 48(3):394-400.

6. Tomazelli JG, Azevedo e Silva G. Rastreamento do câncer de mama no Brasil: uma avaliação da oferta e utilização da rede assistencial do Sistema Único de Saúde no período 2010-2012. Epidemiol Serv Saude 2017; 26(4):713-724.

7. Duarte DAP, Bustamante-Teixeira MT. Iniquidade social e mortalidade por câncer de mama e colo do útero: uma revisão integrativa. Rev Pesqui Cuid Fundam 2018; 10(3):877-888.

8. Bray F, Ferlay J, Soerjomataram I, Siegel RL, Torre LA, Jemal A. Estatísticas globais sobre câncer 2018: estimativas do GLOBOCAN de incidência e mortalidade em todo o mundo para 36 casos de câncer em 185 países. CA Cancer J Clin 2018; 68(6):394-424.

9. Brasil. Ministério da Saúde (MS). Instituto Nacional de Câncer José Alencar Gomes da Silva (INCA). Estimativa 2018: incidência de câncer no Brasil. Rio de Janeiro: INCA; 2017

10. Meira KC, Guimarães RM, Santos J, Cabrelli R. Análise de efeito idade-período-coorte na mortalidade por câncer de mama no Brasil e regiões. Rev Panam Salud Publica 2015; 37(6):402-408.

11. Victora CG, Barreto ML, Leal MC, Monteiro CA, Schmidt MI, Paim J, Bastos FI, Almeida C, Bahia L, Travassos C, Reichenheim M, Barros FC. Health conditions and health-policy innovations in Brazil: the way forward. Lancet 2011; 377(9782):2042-2053.

12. Boccolini CS, de Souza Junior PRB. Inequities in healthcare utilization: results of the Brazilian National Health Survey, 2013. Int J Equity Health 2016; 15(1):150.

13. Silva MJS; Lima FLT; O'Dwyer G; Osorio-de-Castro CGS. Política de Atenção ao Câncer no Brasil após a Criação do Sistema Único de Saúde. Rev Bras Cancerol 2017; 63(3):177-187.

14. Brasil. Ministério da Saúde (MS). Portaria no 2.439, de 5 de dezembro de 2005. Institui a Política Nacional de Atenção Oncológica: Promoção, Prevenção, Diagnóstico, Tratamento, Reabilitação e Cuidados Paliativos, a ser implantada em todas as unidades federadas, respeitadas as competências das três esferas de gestão. Diário Oficial da União 2005; 8 dez. 
15. Brasil. Ministério da Saúde (MS). Portaria no 4.279, de 30 dezembro de 2010. Estabelece Diretrizes para a Organização da Rede de Atenção à Saúde no Âmbito do Sistema Único de Saúde (SUS). Diário Oficial da União 2010; $30 \mathrm{dez}$.

16. Brasil. Ministério da Saúde (MS). Portaria GM no 874, de 16 de maio de 2013. Institui a Política Nacional para a Prevenção e Controle do Câncer na Rede de Atenção à Saúde das Pessoas com Doenças Crônicas no âmbito do Sistema Único de Saúde (SUS). Diário Oficial da União 2013, 29 mai.

17. Santos L, Campos GW de S. SUS Brasil: a região de saúde como caminho. Saude e Soc 2015; 24(2):438446.

18. Chueiri PS, Harzheim E, Gauche H, Vasconcelos LLC. Pessoas com doenças crônicas, as redes de atenção e a Atenção Primária à Saúde. Divulgação em saúde para debate 2014; 52:114-124.

19. Brasil. Ministério da Saúde (MS). Instituto Nacional de Câncer José Alencar Gomes da Silva (INCA). Diretrizes para a deteç̧ão precoce do câncer de mama no Brasil. Rio de Janeiro: INCA; 2015.

20. Traldi MC, Galvão P, Morais SS, Fonseca MRCC. Demora no diagnóstico de câncer de mama de mulheres atendidas no Sistema Público de Saúde. Cad Saude Colet 2016; 24(2):185-191.

21. Migowski A, Azevedo e Silva G, Dias MBK, Diz MDLE, Sant'Ana DR, Nadanovsky P. Diretrizes para detecção precoce do câncer de mama no Brasil. II - Novas recomendações nacionais, principais evidências e controvérsias. Cad Saude Publica 2018; 34(6):e00074817.

22. Silva RCF. Evidências científicas e análise comparada de programas de rastreamento: elementos para a discussão das condições essenciais para o rastreamento organizado do câncer de mama no Brasil [tese]. Rio de Janeiro: Escola Nacional de Saúde Pública Sergio Arouca; 2012.

23. Migowski A, Atty ATM, Tomazelli JG, Dias MBK, Jardim BC. A atenção oncológica e os 30 anos do Sistema Único de Saúde. Rev Bras Cancerolog 2018; 64(2):247250.

24. Oliveira EXG, Melo ECP, Pinheiro RS, Noronha CP, Carvalho MS. Acesso à assistência oncológica: mapeamento dos fluxos origem-destino das internações e dos atendimentos ambulatoriais. O caso do câncer de mama. Cad Saude Publica 2001; 27(2):317-326.

25. Kessner DM, Kalk CE, Singer J. Assessing health quality: the case for tracers. N Engl J Med 1973; 288(4):189194.

26. AcesSUS. Etapa qualitativa, contexto São Paulo [internet]. 2009 Set [acessado 2019 Abr 12]. Disponível em: https://www.fcm.unicamp.br/acessus/metodologia/ etapa-qualitativa/contexto-sao-paulo

27. AcesSUS. Etapa qualitativa, contexto Campinas [internet]. 2009 set. [acessado 2019 Abr 12]. Disponível em: https://www.fcm.unicamp.br/acessus/metodologia/ etapa-qualitativa/contexto-campinas

28. Goldstein H. Multilevel statistical models. London: Arnold; 2003

29. Instituto Brasileiro de Geografia e Estatística (IBGE). Censo demográfico 2010. Rio de Janeiro: IBGE, 2012.
30. Fundação Sistema Estadual de análise de dados (Seade). Disponível em: http://www.seade.gov.br/

31. Brasil. Ministério da Saúde (MS). Instituto Nacional de Câncer José Alencar Gomes da Silva (INCA). A situação do câncer de mama no Brasil: síntese de dados dos sistemas de informação. Rio de Janeiro: INCA; 2019.

32. Lages RB, Oliveira GP, Filho VMS, Nogueira FM, Teles JBM, Vieira SC. Desigualdades associadas à não realização de mamografia na zona urbana de Teresina-Piauí-Brasil, 2010-2011. Rev Bras Epidemiol 2012; 15(4):737-747.

33. Cabral ALLV, Giatti L, Casale C, Cherchiglia ML. Vulnerabilidade social e câncer de mama: diferenciais no intervalo entre o diagnóstico e o tratamento em mulheres de diferentes perfis sociodemográficos. Cien Saude Colet 2019; 24(2):613-622.

34. Medeiros GC, Bergmann A, Aguar SS, Thuler LCS. Análise dos determinantes que influenciam o tempo para o início do tratamento de mulheres com câncer de mama no Brasil. Cad Saude Publica 2015; 31(6):1269-1282.

35. Nogueira MC, Guerra MR, Cintra JRD, Corrêa CSL, Fayer VA, Bustamante-Teixeira MT. Racial disparity in 10-year breast cancer survival: A mediation analysis using potential responses approach. Cad Saude Publica 2018; 34(9):e00211717.

36. Barros AF, Araújo JM, Nascimento CM, Dias A. Itinerário terapêutico de mulheres com câncer de mama tratadas no Distrito Federal, Brasil. Rev Saude Publica [Internet]. [cited 2019 Aug 25]; 53(14): [about 11 p.]. Available from: http://www.scielo.br/pdf/rsp/v53/ pt_1518-8787-rsp-53-14.pdf

37. Ohl ICB, Ohl RIB, Chavaglia SRR, Goldman RE. Ações públicas para o controle do câncer de mama no Brasil: revisão integrativa. Rev Bras Enferm 2016; 69(4):793-803.

38. Arruda RL, Teles ED, Machado NS, Oliveira FJF, Fontoura IG, Ferreira AGN. Prevenção do câncer de mama em mulheres atendidas em Unidade Básica de Saúde. Rev Rene 2015; 16(2):143-149.

39. Marinho LAB, Costa-Gurgel MS, Cecatti JG, Osis MJD. Conhecimento, atitude e prática do auto-exame das mamas em centros de saúde. Rev Saúde Publica 2003; 37(5):576-582.

40. Teixeira MS, Goldman RE, Gonçalves VCS, Gutiérrez MGR, Figueiredo EN. Atuação do enfermeiro da atenção primária no controle do câncer de mama. Acta Paul Enferm 2017; 30(1):1-7.

41. Oliveira EXG, Pinheiro RS, Melo ECP, Carvalho MS Condicionantes socioeconômicos e geográficos do acesso à mamografia no Brasil, 2003-2008. Cien Saude Colet 2011; 16(9):3649-3664.

42. Ramos ACV, Alves LS, Berra TZ, Popolin MP, Arcoverde MAM, Campoy LT, Martoreli-Júnior JF, Lapão LV, Palha PF, Arcêncio RA. Estratégia Saúde da Família, saúde suplementar e desigualdade no acesso à mamografia no Brasil. Rev Panam Salud Publica 2018; 42:e166. 
43. Brasil. Lei $n^{\circ} 11.664$, de 29 de abril de 2008. Dispõe sobre a efetivação de ações de saúde que assegurem a prevenção, a detecção, o tratamento e o seguimento dos cânceres do colo uterino e de mama, no âmbito do Sistema Único de Saúde - SUS. Diário Oficial da União 2008; 30 abr.

44. Tesser CD, D'Avila TLC. Por que reconsiderar a indicação do rastreamento do câncer de mama? Cad Saude Publica 2016; 32(5):e00095914.

45. Silva RCF, Hortale VA. Rastreamento do câncer de mama no Brasil: quem, como e por quê? Rev Bras Cancerolog 2012; 58(1):67-71.

46. Cunha EM, Giovanella L. Longitudinalidade/continuidade do cuidado: identificando dimensões e variáveis para a avaliação da Atenção Primária no contexto do sistema público de saúde brasileiro. Cien Saude Colet 2011; 16(1):2029-1042.

47. Silva MRF, Braga JPR, Moura JFP, Lima JTO. Continuidade assistencial a mulheres com câncer de colo de útero em redes de atenção à saúde: estudo de caso, Pernambuco. Saúde Debate 2016; 4(110):107-119.

48. Santos FPA, Acioli S, Rodrigues VP, Machado JC, Souza MS, Couto TA. Práticas de cuidado da enfermeira na Estratégia Saúde da Família. Rev Bras Enferm 2016; 69(6):1124-1131.

49. Aguiar FAR, Sousa TC, Branco JGC, Costa FBC, Torres ARA, Arruda LP. Produção do cuidado na rede de atenção ao câncer de mama: revisão integrativa. Sanare 2018; 17(1):84-92.

50. Pinto NRS, Tanaka OU, Spedo SM. Política de saúde e gestão no processo de (re)construção do SUS em município de grande porte: um estudo de caso de São Paulo, Brasil. Cad Saude Publica 2009; 25(4):927-938.

51. L'abbate S. Direito a saúde: da reivindicação a realização; projetos de política de saúde em Campinas [tese]. São Paulo: Universidade de São Paulo; 1990.

52. Carvalho SR, Campos GWS. Modelos de atenção à saúde: a organização de Equipes de Referência na rede básica da Secretaria Municipal de Saúde de Betim, Minas Gerais. Cad Saude Publica. 2000; 16(2):507-515.

53. Campos GWS. A clínica do sujeito: por uma clínica reformulada e ampliada. In: Campos GWS. Saúde Paidéia. São Paulo: Hucitec; 2003. p.51-67.

54. Campos GWS. Saúde Paidéia. São Paulo: Hucitec; 2003.

Artigo apresentado em 15/10/2019

Aprovado em 25/11/2020

Versão final apresentada em 27/11/2020

Editores-chefes: Romeu Gomes, Antônio Augusto Moura da Silva 